\title{
ON THE VIBRATIONS OF A CLAMPED PLATE UNDER TENSION*
}

BY

\author{
ALEXANDER WEINSTEIN AND WEI ZANG CHIEN \\ Department of Applied Mathematics, University of Toronto
}

The object of the present paper is the computation of the fundamental frequency of a vibrating clamped square plate under uniform tension. It will be seen that the method used here reduces our problem for a plate of any shape to the membrane problem for the corresponding domain. For this reason similar numerical results could be obtained for a number of other shapes. A similar question has been discussed for a circular plate by W. G. Bickley in connection with the problem of reception of acoustic signals in a condenser microphone. The circular plate is an elementary problem from the theoretical viewpoint. However, the actual calculations involving Bessel's functions are rather heavy. Bickley was able to give the frequencies only for a small range of the tension.

The frequencies of a square plate cannot be obtained explicitly in terms of elementary functions. However, the Rayleigh-Ritz method yields an upper bound for these quantities. The result cannot be considered as satisfactory since this method does not give us an estimation of the error. Fortunately, an increasing sequence of lower bounds can be obtained for all frequencies by the application of a variational method already introduced by one of the authors in several vibration and buckling problems. Combining these lower bounds with the upper bounds obtained by Rayleigh-Ritz, we obtain a narrow interval in which our frequencies are located.

Moreover, it is obvious that for questions like that of microphone reception, the lower bounds are the more important data.

The theory of the new variational method has been developed in several papers. ${ }^{2}$ The modifications in the present case are slight. For this reason we will omit all theoretical details. The reader can easily reconstruct the proofs of the rules which we are following here.

Let $S$ be the domain of a plate of arbitrary shape, and let $C$ denote its boundary. In the numerical applications we shall assume that $S$ is the square $-\pi / 2 \leqq x, y \leqq \pi / 2$.

We denote by:

$2 h$, the thickness of the plate

$T$, the tension

* Received Dec. 11, 1942.

- 'W. G. Bickley, Phil. Mag. (7), 15, 776-797 (1933).

${ }^{2}$ A. Weinstein, Mémorial des Sciences Mathématiques, No. 88, 1937; A. Weinstein, Portugaliae Mathematica 2, 36 (1941). 
$E$, Young's modulus

$\sigma$, Poisson's ratio

$\rho$, density of the material

$D=2 E h^{3} / 3\left(1-\sigma^{2}\right)$, the flexural rigidity

$\omega$, the eigenfrequency (number of pulsations in $2 \pi$ seconds)

$w$, the transversal displacement.

We put $\tau=T / D$. Our problem admits an infinite sequence of eigenfrequencies $\omega=\omega_{1}, \omega_{2}, \cdots$, in place of which we shall use the eigenvalues $\lambda=\lambda_{1}, \lambda_{2}, \cdots$, where

$$
\lambda=\frac{2 h \rho \omega^{2}}{D} .
$$

The displacements corresponding to these eigenvalues will be denoted by $w=w_{1}, w_{2}, \cdots$. These transverse displacements $w$ satisfy in $S$ the differential equation:

$$
\Delta \Delta w-\tau \Delta w-\lambda w=0
$$

with the boundary conditions

on $C$.

$$
\begin{gathered}
w=0 \\
d w / d n=0
\end{gathered}
$$

The equation (1) may be written as follows:

$$
(\Delta+\alpha)(\Delta-\beta) w=0, \quad(\alpha>0, \beta>0)
$$

with

or

$$
\beta-\alpha=\tau, \quad \alpha \beta=\lambda .
$$

$$
\alpha=-\frac{\tau}{2}+\sqrt{\frac{\tau^{2}}{4}+\lambda}, \quad \beta=\frac{\tau}{2}+\sqrt{\frac{\tau^{2}}{4}+\lambda .}
$$

We see that we have the identity:

$$
w=u+\bar{u} \text { in } S+C,
$$

where $u$ and $\bar{u}$ are solutions of

$$
\begin{aligned}
& \Delta u+\alpha u=0, \\
& \Delta \bar{u}-\beta \bar{u}=0 .
\end{aligned}
$$

We have therefore also the following identity:

$$
\Delta w=\Delta(u+\bar{u})=\beta \bar{u}-\alpha u \text { in } S+C .
$$

The identities (7) and (10) will be useful in the following. 
It is well known that the eigenvalues of our plate can be defined by minima problems, the same as could be used in the Rayleigh-Ritz method. For instance, the fundamental eigenvalue $\lambda=\lambda_{1}$, in which we are interested in this paper, is given by the variational problem:

$$
\begin{aligned}
U(w) & \equiv \iint_{S}(\Delta w)^{2} d x d y+\tau \iint_{S}\left[\left(\frac{\partial w}{\partial x}\right)^{2}+\left(\frac{\partial w}{\partial y}\right)^{2}\right] d x d y \\
& =\min =\lambda_{1}
\end{aligned}
$$

with the condition

$$
H(w) \equiv \iint_{S} w^{2} d x d y=1
$$

and with the boundary conditions (2) and (3).

Let us note that $U$ is not the potential energy of the plate. Nevertheless our variational problem gives us the correct differential equation and boundary conditions. This variational problem will be denoted by $P$. The higher eigenvalues $\lambda_{2}, \lambda_{3}, \cdots$ can also be defined by similar variational problems. However, we shall not use them in this paper.

The Euler equation of $P$ is the equation (1). This equation together with the boundary conditions (2) and (3) defines a differential eigenvalue problem $\bar{P}$ which admits the solutions $w_{1}, w_{2}, \cdots$ corresponding to the eigenvalues $\lambda_{1}, \lambda_{2}, \cdots$.

In order to obtain an increasing sequence of lower bounds for $\lambda_{1}$ we begin by cancelling in the variational problem $P$ the boundary condition $d w / d n=0$. In this way we obtain a new variational problem $P_{0}$ :

$$
U(w)=\min =\lambda_{1}^{(0)} ; \quad H(w)=1
$$

with the boundary condition $w=0$.

The conditions in $P_{0}$ being less restrictive than in $P$, we have $\lambda_{1}^{(0)} \leqq \lambda_{1}$. The Euler equation in $P_{0}$ is the same as in $P$, namely the equation (1). However, the boundary conditions for this equation are

$$
w=0 \text { and } \Delta w=0 \text { on } C,
$$

the last condition being a so-called natural boundary condition, i.e., a condition which is automatically satisfied by the minimizing function in $P_{0}$. The corresponding differential eigenvalue problem $\bar{P}_{0}$ is given by (1) and (14). $\bar{P}_{0}$ admits a sequence of eigenvalues $\lambda_{1}^{(0)}, \lambda_{2}^{(0)}, \lambda_{3}^{(0)}, \cdots$ the smallest of which is identical with the minimum $\lambda_{1}^{(0)}$ in $P_{0}$. In the case of a square plate, $\bar{P}_{0}$ is identical with the problem of the vibrations of a supported plate under tension. The problem $\bar{P}_{0}$ can be solved, for a domain of any shape, in terms of the membrane problem for the same domain, a fact which has been implicitly used in the elementary theory of a square supported plate. 
In order to show this we use the identities (7) and (10) and we obtain from (14) at once

$$
u=0, \bar{u}=0 \text { on } C .
$$

In view of (8) and (9), it follows immediately that $\bar{u} \equiv 0$ in $S+C$ and that (1) and (14) are satisfied by $w=u$, where $u$ is an eigenfunction of the membrane problem $\Delta u+\alpha u=0$ in $S, u=0$ on $C$. From the eigenvalues $\alpha$ of this problem we can compute the eigenvalues $\lambda^{(0)}$ in $\bar{P}_{0}$ by using the equations (5).

In order to obtain an increasing sequence of lower bounds for the eigenvalue $\lambda_{1}$ in $P$ we link $P_{0}$ with $P$ by a chain of intermediate variational problems $P_{1}, P_{2}, \cdots$, the solutions of which can be expressed in terms of the solutions of $\bar{P}_{0}$. In this way our problem for the clamped plate can be reduced to the solution of $\bar{P}_{0}$ which is, as we have seen, equivalent to the problem of a vibrating membrane.

In order to show how this can be done, let

$$
p_{1}(s), p_{2}(s), \cdots, p_{m-1}(s), p_{m}(s), \cdots
$$

be an arbitrarily given sequence of functions defined on the boundary $C$ of the plate, $s$ being the arc, and $p_{1}(s)$ being positive. The problem $P_{m}$ $(m=1,2, \cdots)$ is then defined as follows:

Problem $P_{m}$ : Find the minimum $\lambda_{1}^{(m)}$ of $U(w)$ with the condition $H(w)=1$ and with the boundary conditions

$$
\begin{gathered}
w=0 \text { on } C, \\
\int_{C} p_{k} \frac{d w}{d n} d s=0, \quad k=1,2, \cdots, m .
\end{gathered}
$$

The conditions in $P_{m}$ are more restrictive than those in $P_{m-1}$ but they are less restrictive than the boundary conditions in $P$.

We have therefore $\lambda_{1}^{(0)} \leqq \lambda_{1}^{(1)} \leqq \lambda_{1}^{(2)} \leqq \cdots \leqq \lambda_{1}$. The minimizing function $w$ in $P$ satisfies the same Euler equation (1) as in $P_{0}$ (or in $P$ ), but the boundary conditions are given now by the equations (16), (17) and by

$$
\Delta w=a_{1} p_{1}+\cdots+a_{m} p_{m} \text { on } C,
$$

the last condition being again a natural boundary condition. The constant coefficients $a_{1}, \cdots, a_{m}$ are unknowns. In order to solve $P_{m}$ we have to determine the lowest eigenvalue of the differential problem $\bar{P}_{m}$ defined by (1), (16), (17) and (18). We use again the identities (7) and (10) in a way described in our previous papers. In order to avoid repetitions which would considerably increase the length of this paper, we will only formulate the rules for the computation. It can be shown that $\lambda_{1}^{(m)}$ can be computed by the following procedure. Denote by $u_{i}$ and $\bar{u}_{i}(i=1, \cdots, m)$ the solutions of the equations 


$$
\begin{aligned}
& \Delta u_{i}+\alpha u_{i}=0 \\
& \Delta \bar{u}_{i}-\beta \bar{u}_{i}=0
\end{aligned}
$$

with the boundary conditions

$$
\begin{aligned}
& u_{i}=-p_{i}(s) \\
& \bar{u}_{i}=p_{i}(s)
\end{aligned}
$$

where $\alpha$ and $\beta$ are considered as parameters. These equations can be solved in terms of the solutions of $\bar{P}_{0}$. Put $w_{i}=u_{i}+\bar{u}_{i}$ and compute the quantities

$$
\alpha_{i j}(\lambda)=\int_{C} p_{i} \frac{d w_{j}}{d n} d s ; \quad i, j=1, \cdots, m,
$$

where the parameter $\lambda$ is defined in terms of $\alpha$ and $\beta$ by (5) and (6). Then $\lambda_{1}^{(m)}$ is the smallest root of the determinant equation

$$
\left\|\alpha_{i j}(\lambda)\right\|=0 ; \quad i, j=1, \cdots, m
$$

provided that the smallest root is smaller than the second eigenvalue $\lambda_{2}^{(0)}$ of the differential eigenvalue problem $\bar{P}_{0}$, defined by the equations (1) and (14).

The calculation of (23) can be further simplified by introducing a sequence of harmonic functions

$$
p_{1}(x, y), p_{2}(x, y), \cdots, p_{m-1}(x, y), p_{m}(x, y), \cdots
$$

whose boundary values are given as in (15). Then by Green's theorem, (23) can be written as follows

$$
\alpha_{i j}(\lambda)=\iint_{S} p_{i}(x, y)\left(\beta \bar{u}_{i}-\alpha u_{j}\right) d x d y .
$$

Calculation for a square plate $(-\pi / 2 \leqq x, y \leqq \pi / 2)$ : In this case, we take

$$
\begin{aligned}
p_{i}(x, y) & =\frac{\cosh \left(\beta_{2 i-1} \pi / 2\right) \cosh \left(\alpha_{2 i-1} \pi / 2\right)}{\cosh (2 i-1) \pi / 2} \\
1 & \cdot\{\cosh (2 i-1) x \cos (2 i-1) y+\cosh (2 i-1) y \cos (2 i-1) x\}
\end{aligned}
$$

where

$$
\alpha_{2 i-1}=\sqrt{(2 i-1)^{2}-\alpha}, \quad \beta_{2 i-1}=\sqrt{(2 i-1)^{2}+\beta} .
$$

On the boundary, we have

$$
\left\{\begin{array}{l}
p_{i}( \pm \pi / 2, y)=\cos (2 i+1) y \cosh \beta_{2 i-1} \pi / 2 \cosh \alpha_{2 i-1} \pi / 2 \\
p_{i}(x, \pm \pi / 2)=\cos (2 i-1) x \cosh \beta_{2 i-1} \pi / 2 \cosh \alpha_{2 i-1} \pi / 2
\end{array}\right.
$$

Then the solutions of the problems (19), (21) and (20), (22) are 
$\left\{\begin{array}{l}u_{i}=-\cosh \beta_{2 i-1} \pi / 2\left[\cos (2 i-1) x \cosh \alpha_{2 i-1} y+\cos (2 i-1) y \cosh \alpha_{2 i-1} x\right] \\ \bar{u}_{i}=\cosh \alpha_{2 i-1} \pi / 2\left[\cos (2 i-1) x \cosh \beta_{2 i-1} y+\cos (2 i-1) y \cosh \beta_{2 i-1} x\right] .\end{array}\right.$

Substituting $p_{i}(x, y)$ from (27), $u_{i}$ and $\bar{u}_{i}$ from (30) into (26), we obtain after a little calculation,

$\alpha_{i j}=4 \cosh \alpha_{2 i-1} \pi / 2 \cosh \alpha_{2 j-1} \pi / 2 \cosh \beta_{2 i-1} \pi / 2 \cosh \beta_{2 j-1} \pi / 2\left(A_{i j}+B_{i j}\right)$

where

$$
\begin{aligned}
& A_{i j}=A_{j i}=\frac{2(2 j-1)(2 i-1)(-1)^{i+j}}{\left[(2 i-1)^{2}+(2 j-1)^{2}\right]}\left[\frac{\beta}{\beta_{2 j-1}^{2}+(2 i-1)^{2}}+\frac{\alpha}{\alpha_{2 j-1}^{2}+(2 i-1)^{2}}\right] \\
& \left\{\begin{array}{l}
B_{i i}=\frac{1}{2}\left[\beta_{2 i-1} \pi \tanh \beta_{2 i-1} \pi / 2-\alpha_{2 i-1} \pi \tanh \alpha_{2 i-1} \pi-1 / 2\right] \\
B_{i j}=0 \text { for } i \neq j .
\end{array}\right.
\end{aligned}
$$

It should be noted that the roots of $\left\|\alpha_{i j}(\lambda)\right\|$ are equal to the roots of $\left\|\bar{\alpha}_{i j}(\lambda)\right\|$, where

$$
\bar{\alpha}_{i j}=A_{i j}+B_{i j}
$$

The results of our numerical computations are given in Table I below. The first and second columns give $\lambda_{1}^{(0)}$ and $\lambda_{2}^{(0)}$ for the supported plate. The

\begin{tabular}{|c|c|c|c|c|c|c|}
\hline \multicolumn{3}{|c|}{ Supported Plate } & \multicolumn{4}{|c|}{ Clamped Plate } \\
\hline$\tau$ & $\begin{array}{l}\text { 1st eigen- } \\
\text { value } \lambda_{1}^{(0)}\end{array}$ & $\begin{array}{l}\text { 2nd eigen- } \\
\text { value } \lambda_{2}^{(0)}\end{array}$ & $\lambda_{1}^{(1)}$ & $\lambda_{1}^{(2)}$ & $\lambda_{1}^{(3)}$ & $\begin{array}{l}\text { Rayleigh- } \\
\text { Ritz method }\end{array}$ \\
\hline 5 & 14 & 50 & 24.982 & 25.222 & 25.236 & 25.509 \\
\hline 10 & 24 & 75 & 36.639 & 36.845 & 36.862 & 37.443 \\
\hline 15 & 34 & 100 & 48.084 & 48.253 & 48.284 & 49.261 \\
\hline 20 & 44 & 125 & 59.289 & 59.452 & 59.491 & 61.008 \\
\hline 30 & 64 & 175 & 81.651 & 81.760 & 81.809 & 84.372 \\
\hline 50 & 104 & 275 & 125.43 & 125.56 & 125.59 & 130.85 \\
\hline 100 & 204 & 525 & 225.56 & 225.63 & 225.65 & 246.58 \\
\hline 200 & 404 & 1025 & 443.15 & 443.24 & 443.25 & 477.58 \\
\hline
\end{tabular}
next three columns give the smallest root of the determinantal equation (24)

TABLE I

for $m=1,2,3$. Since these roots are smaller than the corresponding second eigenvalues $\lambda_{2}^{(0)}$, they are, according to the general theory, identical with the eigenvalues $\lambda_{1}^{(1)}, \lambda_{1}^{(2)}, \lambda_{1}^{(3)}$ and give therefore an increasing sequence of lower bounds for the fundamental eigenvalue $\lambda_{1}$ in $P$. The corresponding upper bounds, obtained by the Rayleigh-Ritz method are tabulated in the last column. They have been obtained from the variational problem $P$ by putting

$$
w=A \cos ^{2} x \cos ^{2} y+B \cos ^{3} x \cos ^{3} y .
$$


A comparison with $\lambda_{1}^{(3)}$ shows us that the error in the values of $\lambda_{1}$ is, for small tensions, less than 1.2 per cent and, for great tensions, less than 7 per cent. The fact that $\lambda_{1}^{(3)}$ hardly exceeds $\lambda_{1}^{(2)}$ makes it probable that the lower bounds are much closer to the true value of $\lambda_{1}$ than the upper bound given by the Rayleigh-Ritz method.

In figure 1 are plotted curves of funda mental eigenvalues of clamped circular plate and square plate against the tension $\tau$. The curve $I$ is the values of $\lambda_{1}^{(3)}$ for the clamped square plate. The curves II and III are respectively the fundamental eigenvalues for a circular plate of equal area and equal circumference as the given square plate. Both of the latter curves are calculated from the Bickley result.

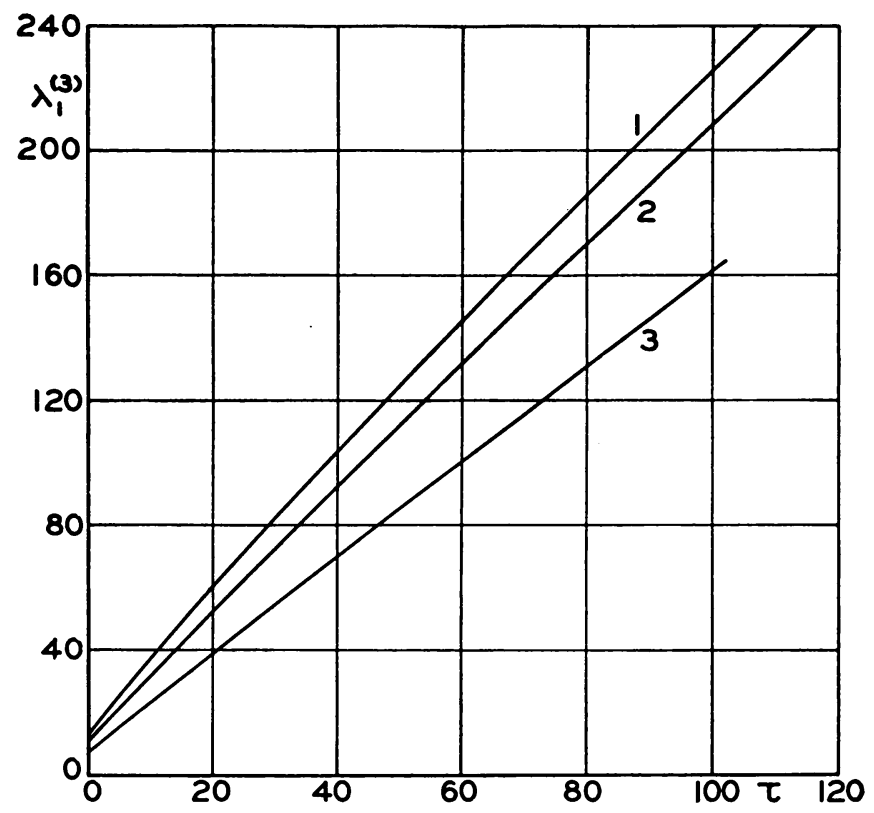

FIG. 1. Curve 1: Clamped Square Plate $(\pi / 2 \geqq x, y \geqq-\pi / 2)$ Curve 2: Clamped Circular Plate $(r=\sqrt{\pi})$

Curve 3: Clamped Circular Plate $(r=2)$

Remark. Using our lower bound $\lambda_{1}^{(3)}$ for a single value $\tau_{0}$ of $\tau$ we can easily compute lower bounds for $\lambda_{1}$ for every value of $\tau$. This result can be obtained by combining our method with an idea of $\mathrm{R}$. V. Southwell. ${ }^{3}$ In fact, the lowest eigenvalue $\lambda_{1}=\lambda_{1}(\tau)$ is given by the minimum of $U(w) / H(w)$ under the con-

${ }^{3}$ H. Lamb and R. V. Southwell, Proc. Royal Soc. Ser. A, 99, 272 (1921). 
ditions (2) and (3). Denoting in (11) the first and second integrals by $J(w)$ and $D(w)$ respectively we have

$$
U / H=J / H+\tau D / H=J / H+\tau_{0} D / H+\left(\tau-\tau_{0}\right) D / H .
$$

Since $D(w) / H(w)$ is obviously greater than 2 (i.e., greater than the lowest eigenvalue of the vibrating membrane) we have for all values $\tau>\tau_{0}$

$$
\lambda_{1}(\tau)>\lambda_{1}\left(\tau_{0}\right)+2\left(\tau-\tau_{0}\right)>\lambda_{1}^{(3)}\left(\tau_{0}\right)+2\left(\tau-\tau_{0}\right) .
$$

Putting $\tau_{0}=5$ we give in Table II the values of $\lambda_{1}^{(3)}(5)+2(\tau-5)$.

TABLE II

\begin{tabular}{rc}
$\tau$ & $\lambda_{1}^{3}(5)+2(\tau-5)$ \\
\hline 5 & 25.236 \\
10 & 35.236 \\
15 & 45.236 \\
20 & 55.236 \\
30 & 75.236 \\
50 & 115.23 \\
100 & 215.23 \\
200 & 415.23
\end{tabular}

It will be seen that these lower bounds for $\lambda_{1}$ are smaller than the lower bounds computed by our method alone. 